\title{
Trayectorias sociales como enfoque para analizar juventudes
}

\author{
Oscar Dávila León ${ }^{1}$ \\ Felipe Ghiardo Soto²
}

\begin{abstract}
RESUMEN
Este trabajo se ocupa de plantear algunas problematizaciones que vayan encaminadas a discutir la pertinencia conceptual y metodológica de las trayectorias sociales como posible enfoque que nos permita «analizar juventudes», asumiendo que la ubicación o espacio donde radican con mayor propiedad las trayectorias sociales es en el campo de la políticas públicas. Se presenta y discuten las nociones más comunes en uso en torno a las trayectorias sociales, con énfasis en las realidades de las juventudes, constatando las potencias, límites y dificultades metodológicas, de técnicas y de fuentes para el desarrollo de la investigación social bajo este enfoque.
\end{abstract}

PALABRAS CLAVE: JUVENTUD, TRAYECTORIAS SOCIALES, CURSOS DE VIDA

1 Asistente Social. Investigador del Centro de Estudios Sociales (cidpa) Valparaíso, Chile. Correo electrónico: oscar@cidpa.cl.

2 Licenciado en Sociología. Investigador del Centro de Estudios Sociales (cidpa) Valparaíso, Chile. Correo electrónico: felipe@cidpa.cl. 


\title{
Trajetórias sociais como abordagem para analisar juventudes
}

\section{RESUMO}

Este artigo tem como objetivo apresentar algumas problematizações que visam discutir a pertinência conceitual e metodológica das trajetórias sociais como possível abordagem que permita "analisar juventudes", assumindo que a localização ou espaço onde as trajetórias sociais estão presentes com maior propriedade é no campo das políticas públicas. São apresentadas e discutida as noções mais comuns em uso em torno das trajetórias sociais, com ênfase nas realidades das juventudes, constatando as potências, limites e dificuldades metodológicas, de técnicas e de fontes para o desenvolvimento da pesquisa social sob esta abordagem.

PALAVRAS - CHAVE: JUVENTUDE, TRAJETÓRIAS SOCIAIS, CURSOS DE VIDA

\section{Social trajectories as an approach to analyze youths}

\begin{abstract}
This work is oriented to state some problematizations leading to discuss on the conceptual and methodological pertinence of social trajectories as a potential approach for "youth analysis", assuming that social trajectories are mostly a matter of the field of public policies. The most extended notions concerning social trajectories are presented and discussed, with an emphasis on the realities of youths, portraying the methodological, technical and source potentialities, limits and difficulties for the development of social research under this approach.
\end{abstract}

KEYWORDS: YOUTH, SOCIAL TRAJECTORIES, LIFE PATHS. 
Nosotros, los de entonces, ya no somos los mismos.

Poema 20, Pablo Neruda

\section{INTRODUCCIÓN}

Intentar «analizar juventudes» en el contexto del Chile contemporáneo ha abierto interesantes discusiones al respecto. Las han habido desde distintos puntos de vista, pero que sin duda las que se visualizan con más potencia y capacidad descriptiva -y en algunos casos, explicativa - han sido las de carácter conceptuales y metodológicas, en su afán de poder situar estos intento dentro de un conjunto de perspectivas teóricas, conceptuales y con las nociones en uso más apropiadas para sus propósitos. Y de su parte y de manera complementaria, se hallan las discusiones y experiencias de investigación en materia de juventud centradas en su dimensión metodológica, sobre la base de enfoques y su correlato instrumental disponible en el campo de las ciencias sociales y la investigación social.

Bajo esos enunciados iniciales, nos interesa continuar la discusión sobre esas materias, las cuales ya llevan un tiempo y cada vez surgen nuevos abordamientos y experiencias de investigación que posibilitan ahondar y probar los rendimientos conceptuales y metodológicos posibles de obtener.

Las trayectorias sociales, trayectorias juveniles, transiciones a la vida adulta, los cursos de vida, las construcciones biográficas, las nuevas condiciones juveniles contemporáneas, la construcción y adquisición de los capitales (sociales, culturales, escolares), entre otras, han sido las nociones con las cuales nos hemos relacionado y trabajado por considerarlas pertinentes y apropiados en el propósito de intentar analizar juventudes a tiempos actuales (cf. Dávila y Ghiardo, 2008a; 20IIc; 20IId; 2012b; 2016; y con Medrano, 2005).

Y desde el punto de vista de los enfoques y técnicas de investigación ha resultado interesante las posibilidades que ofrecen la combinación y complementariedad de aproximaciones metodológicas en el afán de ampliar los marcos comprensivos que nos ofrece el uso de enfoques y fuentes diversas. Es el intento de integrar en los análisis sobre juventudes las miradas de tipo cualitativas y cuantitativas, como una manera de acercamiento hacia las subjetividades e intersubjetividades subyacentes en los sujetos sobre dimensiones relevantes de su experiencia de vida; como a su vez, el diálogo necesario con las «condiciones de posibilidad» a partir de los contextos y situaciones de orden más vinculados con la estructura social, con las dimensiones estructurales que -de una u otra manera- interactúan, favorecen o limitan ciertos horizontes de posibilidades, sean en el plano de las aspiraciones, intereses y expectativas de los sujetos jóvenes.

Por otra parte, en la tarea de analizar juventudes bajo un enfoque de trayectorias sociales aparecen con mayor relevancia para el análisis ciertos pro- 
cesos y sus tematizaciones en los planos de la formación y la reproducción. Siempre vistos estos campos de manera relacionada y en permanente diálogo entre las subjetividades de los sujetos y su relación y ubicación en la estructura y sus relaciones sociales. Por ello, esta dinámica va más allá de sólo ser un esfuerzo de «tematizaciones» de la vivencia juvenil en un determinado período vital y trayecto biográfico, sino que demanda examinar las posibles relaciones posibles de establecer entre ciertos hitos juveniles, o si se quiere, de las vivencias de los jóvenes como componentes de sus trayectorias hacia la vida adulta, conjugando en aquellos los planos de las expectativas con las posibilidades de concreción.

Es un intento de construir, analíticamente y con perspectiva de historicidad, los trayectos dentro de los proyectos de vida juveniles, donde nos encontramos de manera especial con dimensiones propias de la etapa formativa y de la escolarización, el acceso e ingreso al mundo laboral, el emparejamiento y la conformación de familia propia, nupcialidad, la maternidad o paternidad o su ausencia, los proyectos de emancipación residencial, por nombrar quizá los principales y con mayor visibilidad y trascendencia en las trayectorias en este tipo de etapa vital: la juventud.

Estos propósitos, orientados hacia un análisis de juventudes, no se encuentran exentos de dificultades, tanto en sus definiciones conceptuales y sus formas de interrelación entre dimensiones integrantes de sus trayectorias, como desde un punto de vista metodológico, y en especial a ser relevado: la producción de datos cualitativos y cuantitativos, y la disponibilidad de fuentes primarias y secundarias. Si bien es cierto que en Chile ha habido un avance importante en las últimas dos décadas en la producción de datos en materia de juventud y temáticas conexas desde la institucionalidad pública (en especial ciertas encuestas y estudios periódicos, censos de población y vivienda, entre otros), y mejor aún, la firme decisión de la administración por garantizar su disponibilidad y transparencia, al igual que una importante mejora y disponibilidad de los registros administrativos públicos; todavía tenemos deficiencias y limitaciones con estos insumos necesarios para la investigación social en materias de juventud.

Sobre estos tópicos enunciados es que deseamos plantear algunas problematizaciones que vayan encaminadas a discutir la pertinencia conceptual y metodológica de las trayectorias sociales como posible enfoque que nos permita «analizar juventudes».

\section{LOS TRAYECTOS SOCIALES: SUS FUENTES}

Resulta difícil y complejo captar, vía fuentes secundarias, la trayectoria social de los sujetos, su «movilidad» estructural. Las encuestas de caracterización socioeconómica son una alternativa que cubre parte de este problema, pero adolecen de las mismas limitaciones que los otros instrumentos: no informan sobre 
los cambios de condición de un mismo caso entre una medición y otra. Con las encuestas permanentes de hogares esto debiera poder resolverse, pero «a futuro», no «hacia atrás», por el poco tiempo que llevan aplicándose.

Por lo cual ni los censos ni las estadísticas vitales incorporan la dimensión subjetiva de los cursos de vida, las razones, expectativas, disposiciones y habitus, diría Bourdieu (I988), estrategias y principios de decisión que se van poniendo en juego en toda fase en la producción de biografías. La consecuencia es que «condiciones» de hecho y «representaciones» subjetivas permanecen como niveles separados sobre cuyos nexos hay más hipótesis que evidencia empírica.

Solamente para el caso de las generaciones más jóvenes podemos encontrar fuentes de información que permiten ese diálogo. Las Encuestas Nacionales de Juventud, en particular, tienen esa facultad de registrar el momento en que ocurre un hecho -por ejemplo, el término de los estudios o el ingreso al mundo laboral-, las razones - por qué no siguió estudiando o entró a trabajar-, y luego ver cómo este par situación/motivo se distribuye de acuerdo a la condición socioeconómica, el sexo y otras características. También permiten abordar distintas esferas de la vida de los jóvenes en un mismo instrumento - educación, trabajo, sexualidad, relaciones familiares, etc. - y con ello se pueden vincular las distintas esferas o dimensiones que componen las trayectorias de vida para ver cómo se va tejiendo sus itinerarios, ${ }^{3}$ y hasta qué punto sus múltiples combinaciones y secuencias temporales se vinculan o no a determinadas condiciones socioeconómicas, de sexo, edad o lugar de residencia. ${ }^{4}$

El problema con las encuestas nacionales de juventud es que, al igual que los censos de población y las encuestas de Caracterización Socioeconómica Nacional (casen), no permiten seguir la evolución de un mismo caso entre dos o más encuestas, pues los casos no son los mismos. Hay algunas excepciones a este planteo, en cuanto experiencias de estudio que abordan las trayectorias de población joven por la vía del seguimiento de ciertas cohortes sobre la base de registros administrativos públicos. Un buen ejemplo de aquello es el de Larrañaga, Cabezas y Dussaillants (20I3) sobre trayectorias educacionales e inserción laboral de estudiantes de la enseñanza media técnico profesional.

Lo que sí rescatamos es que este tipo de análisis recién reseñado, que permite ir tejiendo relaciones entre las esferas de las biografías, describirlas en sus propias temporalidades sin perder la juntura entre razones internas y condicionantes estructurales externas.

Es bajo esa modalidad que sería posible ir avanzando en este tipo de análisis, pudiendo incluso hacerse extensivo al conjunto de la población y a la

3 Ver, por ejemplo, a qué edad terminaron los estudios, de qué tipo de estudios se trataba, si significó o no ingresar inmediatamente al trabajo, a qué tipo de trabajo, si esto se dio en paralelo a la formación de pareja y un hogar independiente o no.

4 Un ejemplo de este tipo de análisis con encuestas nacionales de juventud se puede encontrar en Dávila y Ghiardo (2008a). 
totalidad de sus cursos de vida, más allá de las juventudes. Un trabajo de este tipo tiene amplios alcances por lo siguiente: las transformaciones estructurales que vienen desde el impulso desarrollista/modernizador hasta la reestructuración neoliberal y su transfiguración posterior a economía social de mercado, han tenido como correlato una serie de cambios en el modo en que los sujetos construyen sus vidas.

El carácter de estos cambios ha sido tratado por diversos autores (cf. Garretón, I994) de cuyos trabajos se desprende que entender la estructura y el sentido de estos procesos obliga a vincular multiplicidad de ámbitos, desde la economía a la cultura, asumir que son los sujetos-actores quienes encarnan esos procesos y que lo hacen desde sus particulares posiciones sociales y momentos biográficos.

\section{LAS TRANSICIONES A LA ADULTEZ}

Como tema, los cambios en los modos en que se construyen las biografías ocupan un lugar relevante en el estudio y reflexión acerca de mundos juveniles, particularmente cuando se observan los procesos de «transiciones a la vida adulta». Diversos trabajos revelan la emergencia de nuevas modalidades de construcción de autonomías, ya no lineales y «formateadas» como hace algunas décadas, sí más flexibles e inciertas, que no serían sino una de las formas en que se plasman los procesos de transformación social ocurridos en las últimas décadas (Machado Pais, 2002a; Casals et. al. 2006; Van de Velde, 2005).

En la «sociedad de la información» la exigencia de habilidades cognitivas sería tal que la más clara si no la única posibilidad para que los sectores ya posicionados mantengan su posición y los sectores desventajados aspiren a resolver su desventaja es estudiando, especializándose, capacitándose (Torche y Wormald, 2004). Traducido a trayectoria de vida, este imperativo genera aumentos transversales en la participación escolar de todos los sectores y una extensión del período de estudios respecto a generaciones anteriores. No obstante, como demuestran estudios en el campo educacional, el «capital escolar heredado» actúa sobre las desventajas educativas y así la sociedad que segregaba por desigualdades de acceso al sistema transfiere su pasado al presente para convertirse en otra que ahora segrega por la acción del sistema educacional mismo (Cox, 2003; Redondo, 2005).

La otra cara de lo que ocurre con la escolaridad aparece en el mundo laboral, pues así como se alarga el proceso de escolarización, se retrasa el ingreso al mundo del trabajo, o se hace más laxo. Es lo que señalan algunos estudios sobre el desempleo juvenil o las nuevas relaciones de los jóvenes con el empleo (cf. Weller, 2006). Como indican algunos estudios de la Dirección del Trabajo, la característica básica del mercado laboral en Chile no es, como se solía pensar, la estabilidad, sino la «certeza de la incertidumbre» (Henríquez y Uribe-Echeverría, 2003), que como condición general, afecta a todos los trabajadores, al 
punto que se instala como «sentido común» frente al trabajo en Chile, especie de «adaptación subjetiva» que «naturaliza» la inestabilidad laboral como parte del «ser de las cosas» (Araujo y Martuccelli, 20I2), pero que se expresa de forma particularmente fuerte entre los más jóvenes.

Lo hace defacto en forma de una tasa de desempleo juvenil que duplica a su homóloga adulta y de unos niveles de rotación que llegan a casi el doble de veces que los adultos (Henríquez y Uribe-Echeverría, 2003). Y lo hace también como subjetividad y praxis, mediante un discurso que «re-significa la estabilidad» como solución subjetiva a la distancia entre las condiciones concretas del mundo del trabajo y las expectativas de realización personal (Dávila y Ghiardo, 2008a), o mediante la adopción de nuevas prácticas de relación con el trabajo, como el no-trabajo que estudia Zilvetti (2009), que bien deslegitima al empleo como mecanismo de movilidad social, legitima una relación instrumental que se concentra en la sola remuneración y subraya, de paso, la distancia respecto a un mundo del trabajo «adulto» que resuena lejano y ajeno. ¿Signo de los tiempos, arreglo pasajero propio de una «edad juvenil» o expresión de la falta de expectativas de futuro de los sectores más precarizados?

Como sea, la suma entre períodos de escolaridad más largos y la transferencia temporal de la estabilidad laboral en un mundo del trabajo inestable tienen como correlato decisiones sexual-reproductivas y de vida afectiva de mujeres y hombres. Separadas sexualidad y reproducción, las formas de la intimidad se modifican, sobre todo entre las mujeres (Giddens, I998; Beck, I998). Nuevas formas de identidad femenina, más autónomas e individualizadas, orientadas al logro de una independencia económica y una autonomía afectiva inseparables de los proyectos laborales y aspiraciones profesionales, terminan implicando postergar la procreación y la vida en pareja y ensayar distintos modos de residencia (Montilva, 2007; 2008). El descenso en las tasas de nupcialidad y los cambios en las formas de convivencia en pareja es una de sus manifestaciones más notorias, y la curva descendente en las tasas de fecundidad, su consecuencia lógica.

Desde la década de los sesenta que viene a la baja, pero en el último tramo llega a niveles por debajo de los de reemplazo, al punto que Chile, junto con Cuba, son actualmente los dos países que ocupan los puntos más bajos de toda América Latina. Vista de cerca, sin embargo, la curva esconde matices. Schkolnik y Chakiel (2004) estudiaron las prácticas reproductivas de diversos países de América Latina y mostraron la segmentación social que recubre el desfase entre grupos «adelantados» y «rezagados» en la reducción de la fecundidad. Pese a la convergencia que muestran respecto a las mujeres de sectores «adelantados»-de estratos medios y altos- en el uso de métodos anticonceptivos, las mujeres de los grupos «rezagados», de sectores con menores recursos, al tiempo que usan con menos frecuencia métodos anticonceptivos, mantienen una nupcialidad temprana que les impide resolver la ecuación entre el número de hijos deseado y el que realmente tienen. Recientemente esto pareciera cam- 
biar, pero sólo en algunos grupos específicos, particularmente entre las mujeres adultas de sectores populares económicamente activas, que muestran una tasa de fecundidad más baja que mujeres de altos ingresos debido, principalmente, a los bajos salarios, desprotección y carencia de soportes institucionales (Villalón y Vera, 2012). Sin embargo, los cambios culturales que caracterizan la «segunda transición demográfica» en Europa y los países desarrollados (Van de Kaa, 1987; Lesthaeghe y Moor, 2000), en sociedades segmentadas siguen adquiriendo la forma de pasos desfasados o principios de acción que parecen antitéticos e irreductibles.

\section{LOS TEJIDOS BIOGRÁFICOS}

La repercusión de estas tendencias estructuralmente diferenciadas extiende su influencia a ámbitos aparentemente tan distantes como las formas de familia, ${ }^{5}$ la sexualidad, la estructuración social y el comportamiento político. Lo que nos interesa destacar con esto son básicamente tres puntos. Primero, la estrecha e inevitable relación entre las distintas esferas o dimensiones de los cursos de vida. Ningún estudio que indague uno de estos campos puede evitar alusiones a lo menos a uno de los otros. Muchos estudios, de hecho, establecen la relación desde el mismo título: «educación y empleo» (Abdala, 2002), «trabajo y reproducción» (Baizán, 2006; De Oliveira y García, 1990), «género y trabajo» (Henríquez et. al., 2004; De Oliveira y García, r99o; Longo, 2009; Palma, 2012). Segundo, cualquier análisis debe dar cuenta de la complejidad que imprime la segmentación social a la forma y devenir de estos procesos. Sólo desde ahí se puede entender cómo el tejido biográfico, sus tiempos y razones difieren entre personas que pertenecen a diferentes clases sociales, sexos y generaciones. Tercero, que para entender la composición actual de la variación entre modalidades de cursos de vida hay que seguir su genealogía, su conformación histórica, y para eso es clave seguir el curso de vida de sujetos individuales y establecer relaciones de continuidad y ruptura entre los cursos de viday las trayectorias sociales de distintas generaciones. Esa es la mejor forma para entender en qué grupos, en qué grado, en qué momento y en qué dirección se dan o no determinados cambios.

Pues si es cierto, por ejemplo, que los sectores de más escasos recursos permanecieron fuera de la escuela, también lo es que hubo fragmentos de estos grupos que de todos modos continuaron estudios más allá de los años que fueron los «típicos» para hombres y mujeres de su tiempo y condición social.

5 En términos agregados, las tendencias apuntan a una disminución de los vínculos maritales y una aumento de las uniones de hecho, familias más reducidas, con menos hijos que antes, aumento de los hogares monoparentales, pero también de los extendidos -donde conviven más de una familia - y de los unipersonales (Arriagada, 2004). Sin embargo, al comparar por quintiles, queda en evidencia que los hogares monoparentales y extendidos se concentran en los quintiles con menores recursos, mientras que los unipersonales llegan a cerca del 15\% en los sectores de altos ingresos y abundancia de profesionales jóvenes. 
¿De qué modo resolvieron las condiciones del entorno para dar ese curso a sus vidas?, ¿qué expectativas pusieron en juego en sus decisiones?, ¿implicó un cambio respecto a la trayectoria escolar/laboral típica para los miembros de su sexo en su grupo familiar de generaciones anteriores? Su trayectoria escolar y laboral, ¿provocó una «movilidad ascendente» que los distingue de sus pares generacionales?, ¿se puede tender, a partir de ahí, lazos intergeneracionales que liguen con las trayectorias educativas y laborales de generaciones posteriores? En el plano laboral, ¿implicó una reconversión del campo de actividad laboral respecto al de sus progenitores? ¿Puede eso informar algo respecto sobre los procesos históricos de estructuración social? ¿Y sobre los cambios en las formaciones familiares? ¿Tiene que ver esto con socavones más o menos profundos en los soportes del patriarcalismo tradicional, con grados diferentes de individuación, con posturas socioepocalmente diferenciadas respecto a la secularización de los referentes normativos? De ser así, estos procesos ¿varían dependiendo de las clases y grupos?, ¿presentan los mismos ritmos o se dieron primero en algunos segmentos que en otros? Y si fuera así ¿en cuáles?

\section{PRODUCCIÓN DE BIOGRAFÍAS}

Cuestiones como éstas son de difícil resolución con las fuentes existentes a tiempos actuales. De allí que se presenta como relevante el imaginar otros instrumentos y técnicas de producción de datos que se orienten y tengan como propósito el reconstruir las distintas variantes generacionales de producción de biografías mediante un ejercicio que vincule los aspectos subjetivos y «objetivos» que hasta ahora se ha tratado de abordar por separado. Como señala Casal et. al. (2006)., si se quiere dar cuenta de los cambios sociales y culturales que cargan las formas emergentes de transiciones a la vida adulta, los estudios cualitativos son relevantes, pero penan estudios cuantitativos que sean capaces de conectar pasado $y$ presente, condiciones objetivasy subjetivas, y un enfoque generacional.

Integrando estos tres niveles se podrá captar la diacronía y la sincronía, el movimiento y las diferencias internas, y aportar un marco, a partir de agrupamientos de «casos» con devenires homólogos -ya no de conjuntos sociodemográficos predefinidos-, para una explicación más completa y matizada de la dinámica entre los diferentes tensiones que cada conjunto generacional ha debido ir resolviendo en sus respectivas trayectorias de vida. Con esto es posible aportar a una línea de investigación que intente abordar los cambios socioculturales a partir de los modos en que los sujetos-actores configuran sus vidas. Algunos países, sobre todo europeos, desde hace un tiempo vienen realizando estudios de este tipo. Incluso hay centros institucionales que coordinan estudios comparados a nivel internacional. ${ }^{6}$ En Chile, sin embargo, las iniciativas

6 Karl Ulrich Mayer, uno de los investigadores más prolíficos en este campo, coordina el «Center 
todavía son muy escasas y aisladas, a pesar de la importancia que investigadores le reconocen tanto para el conocimiento social como para la formulación de políticas públicas (cf. Sepúlveda, 20I0). También vale destacar iniciativas desplegadas por Corica, Otero y Miranda en esa línea de investigación para la realidad de jóvenes argentinos (cf. Corica y Otero, 20I8; Miranda y Corica, 20I8). En esa línea, adquiere pertinencia y relevancia el desarrollar ejercicios retrospectivos, que puedan ayudar al armado de una «línea base» para una investigación más sistemática y regular en el tiempo: sería una forma de encontrar piezas que hasta ahora nos faltan para interpretar con mayor claridad y precisión estos procesos.

\section{LAS NOCIONES EN JUEGO}

Para abordar el tema de los cursos de vida y las trayectorias sociales de distintos conjuntos generacionales, es necesario describir de manera breve estos tres conceptos para una mejor comprensión y aclaración de estas nociones.

Cursos de vida -o trayectorias de vida - es el concepto central de una línea de trabajo que estudia los cambios socioculturales a partir del modo en que las personas construyen sus etapas vitales. Glen Elder fue uno de los primeros en acuñar el concepto en un estudio que siguió durante varios momentos a un conjunto de niños que habían vivido la Gran Depresión Económica en los Estados Unidos de la década del r930 y a un grupo de adolescentes y jóvenes que vivieron la Segunda Guerra Mundial (Elder, 1999). Para su trabajo lo importante era tener claro que entender los procesos vividos por estos niños y jóvenes era necesario partir de la base que los cambios en la experiencia de los sujetos, su experiencia en las distintas dimensiones de la vida, sea el ingreso a la escuela, el paso a una vida laboral más o menos definitiva, la formación de pareja y familia, el hecho de tener o no hijo, son siempre parte de un proceso que se desarrolla en relación a un marco social de referencia que les otorga una forma y un significado distintivo.

Como aclara Karl Ulrich Mayer, si el estudio de cursos de vida exige ir estableciendo conexiones entre los eventos o episodios particulares de las distintas etapas de la vida, resulta fundamental e inevitable plantear estudios de largo plazo, que permitan ir siguiendo a agregados de individuos que hayan experimentado algún acontecimiento dentro de un mismo intervalo de tiempo. De estas cohortes, de preferencia cohortes etarias, importa el modo en que transita por lo que define como dominios de la vida, que incluyen la etapa de estudios, el trabajo, la vida familiar, y cuya forma y encadenamiento resulta de un proceso que combina características personales y la acción individual, así como el marco histórico y sociocultural, los contextos institucionales y las condiciones estructurales en que viven los sujetos (Mayer, 2004).

\footnotetext{
for research on inequalities and the life course», con sede en la Universidad de Yale.
} 
Como señalan Heinz y Krüger, el estudio de cursos de vida, para que sea completo, tiene que descubrir cómo interactúan las condiciones condicionantes que conlleva el hecho de ocupar una posición en la estructura social, las reglas y regulaciones institucionales, con sus soportes y tiempos, y las subjetividades y decisiones de los individuos a lo largo de su vida. Todo lo cual necesariamente requiere considerar el factor tiempo tanto en forma de tiempo histórico o marco epocal como en la forma de temporalidades que asumen las biografías individuales (Heinz y Krüger, 200I).

Juntando esos componentes estructurales, institucionales y de disposiciones subjetivas, el curso de vida queda como una secuencia de etapas cultural e institucionalmente condicionado, que implica la asunción de diversos roles y la ubicación dentro de la estructura social. Este proceso de posicionamiento en la estructura social - el espacio social de Bourdieu (ig88) - es lo que en otros trabajos hemos preferido diferenciar analíticamente y definir como trayectorias sociales (Dávila, Ghiardo y Medrano, 2005; Dávila y Ghiardo, 2008a). El concepto de trayectorias sociales viene de Bourdieu y su intento de complejizar el análisis de los mecanismos que definían la estructura de clases en las sociedades contemporáneas (Bourdieu, I988). Para Bourdieu, la función entre la cantidad y tipos de los distintos tipos de capital que poseen los individuos-agentes define una posición en la estructura social cuyas coordenadas y distribución de posiciones está históricamente circunscrita. Cada posición está asociada a un habitus, a un conjunto de disposiciones subjetivas socialmente construidas y legitimadas que impregnan las prácticas de los sujetos. Para entenderlas es necesario asumir que la distribución presente de capitales es la condensación de la historia de la lucha por la posesión de capitales entre grupos desiguales. Por lo tanto, la trayectoria social viene a ser la secuencia de posiciones que un sujeto va ocupando a lo largo de su vida cuya interpretación deberá tener siempre como referencia posición de origen que no es sino el punto que traduce la trayectoria histórica de generaciones anteriores de una clase. Sólo así se puede establecer si los individuos se ciñen a las fuerzas de enclasamiento o se despegan y desclasan de la trayectoria típica para el conjunto de individuos-agentes que ocupan una misma posición de origen en determinado momento de la distribución socialmente diferenciada de recursos y oportunidades (Bourdieu, I988).

Así entendidas, las trayectorias sociales vienen a ser la cara estructural de los cursos de vida. Mientras el curso de vida observa la secuencia que producen las distintas «fases» o etapas de la vida, las trayectorias sociales observan el trazo que ellas dibujan en el plano de las posiciones estructurales y las disposiciones subjetivas que envuelven y a su vez producen esos cambios de condición. Si para el análisis de las transiciones el paso de estudiante a trabajador importa en sí mismo, si la edad en que se produce es un factor que influye en la descripción, las trayectorias del grupo social de origen, el nivel de educación alcanzado, el tipo de establecimiento escolar, el título y el tipo de trabajo al que se accede con ese título, la valoración social y simbólica del título obtenido. 
Lo que aquí definimos como conjuntos generacionales alude a los colectivos de sujetos que encarnan y a la vez producen los procesos que tratan los cursos de vida y las trayectorias sociales. No son grupos generacionales en el sentido que le da Mannheim al término, porque no refieren a las formas organizadas de sujetos de acción sociopolítica que se asocian por una interpretación compartida de una experiencia histórica común (cf. Mannheim, I982). Son conjuntos en tanto constructo de análisis cuantitativo: individuos que pueden carecer de toda conexión en la vida-real-cotidiana, pero que en la medida que comparten características de edad, clase y sexo, son factibles de agrupar y observar mediante herramientas de investigación cuantitativa. Son más bien como los grupos socioepocales (Canales y Ghiardo, 2012): forman parte de generaciones, del conjunto de sujetos que nace en determinado momento y experimenta los procesos sociohistórico en una misma etapa de sus vidas, pero constituyen los subconjuntos que resultan de la desagregación de una generación tratada en tanto cohorte etaria.

Con estos conceptos como referencia teórico-metodológica se han desarrollado una gran cantidad de estudios. Por citar sólo unos pocos ejemplos, a mediados de los ochenta Fetherman y Sorensen ensayaron un estudio sobre los cambios en las estructuras económicas e institucionales de Noruega sobre la base de una análisis cuantitativo que seguía a tres cohortes de noruegos. Así pudieron medir que la industrialización post-segunda guerra mundial se asociaba claramente a un cambio en la duración de la escolaridad de los jóvenes y en el retardo del ingreso al mercado laboral, al tiempo que detectaba también una creciente estratificación social por edades (Fetherman y Sorensen, 1983).

Desde más o menos los mismos años, Karl Ulrich Mayer viene realizando una serie de trabajos que son ilustrativos de las potencialidad analítica del concepto. Lo aplicó para contrastar los cursos de vida que generan sociedades liberales y, con instituciones y sistemas de protección diferenciados, con economías liberales y socialprotectores y pautas culturales más individualistas como los Estadios Unidos, y socialfamilísticas como las del sur de Europa (Mayer, 2004). El mismo Mayer estudió los cambios que produjo el término del socialismo real en la Alemania oriental sobre las vidas laborales de diferentes generaciones (Mayer et al., 1993). Con la profusión de investigadores se han publicado trabajos colectivos que formalizan el enfoque y exponen sus resultados en múltiples ámbitos (Mortimer y Shanahan, 2003).

\section{A MODO DE COLOFÓN: ANALIZAR JUVENTUDES}

Valga rememorar, como cierre del texto, dos hitos/fechas que alcanza la Revista Última Década con este volumen: su número cincuenta y los veinticinco años desde su primera edición en el año i993. Largo período para una publicación, 
precisamente ocupada de difundir y poner en común las marchas y contramarchas de la investigación y estudios en materias de juventudes, y además, entendida como un espacio de debate y diálogo sobre variados tópicos, a los cuales nos hemos referido. ${ }^{7}$ La referencia a esta publicación guarda relación con las discusiones teóricas, conceptuales y metodológicas sobre las formas y herramientas apropiadas para la definición del sujeto juvenil, o en otras palabras, cómo se ha intentado la construcción social de la categoría juventudes como objeto/sujeto de estudio durante el tiempo más contemporáneo en la realidad chilena.

Es así como planteábamos al comienzo del texto, como interrogante y como desafío, la premisa sobre si las trayectorias sociales podían constituirse en un enfoque pertinente que permitiera «analizar juventudes», entendidas aquéllas como categoría, noción y que a su vez nos refiere a un conjunto amplio de estrategias y enfoques metodológicos, técnicas y fuentes que operan como soporte apropiado para la investigación social en juventud desde un enfoque de trayectorias. Nos queda la convicción, luego del desarrollo de este trabajo y otros anteriores, que de manera efectiva el enfoque y su uso de la noción de trayectorias sociales aporta al conocimiento y análisis de las condiciones juveniles.

Para terminar, y no obstante lo anterior, es preciso realizar dos alcances, los cuales se constituyen también como desafíos para ciertas estrategias conceptuales y metodológicas. La primera, hace referencia a la ubicación o espacio donde radican con mayor propiedad las trayectorias sociales: en el campo de las políticas públicas. Pues no sólo poseen el interés del conocimiento, descripción e intención de explicación de determinados fenómenos que atañen a las juventudes, sino que su énfasis se encuentra en el diálogo y discusión con la política pública en orden a las necesarias mejoras en las condiciones de vida de este grupo etario, sean de tipo materiales e inmateriales, como son los accesos y permanencias en el campo educacional y laboral, pasando por las dimensiones relevantes de la subjetividad, valores sociales, participación, empoderamiento, la cuestión del espacio público, desigualdades sociales, igualdad de género, proyectos de vida, entre muchas otras. Funciones básicas de las trayectorias sociales se relacionan directamente con el conocimiento de las realidades juveniles, el diseño de estrategias de mejora, el seguimiento y monitoreo, la evaluación y reformulación de políticas públicas dirigidas a las juventudes. Este creemos que es una de sus mayores potencias y ubicación privilegiada de las trayectorias sociales en cuanto a propender a «analizar juventudes». La segunda, maridada con la anterior, guarda relación con la inclusión de la dimensión generacional, no sólo la que podamos denominar juventud, sino que la interesante y necesaria comparabilidad con las demás generaciones de la sociedad. Pues las trayectorias sociales incluyen no sólo «el trayecto juvenil». En este campo de actuación investigati-

7 Para un profundo y exhaustivo análisis y discusión sobre contenidos, temas y trabajos publicados en la revista, ver Duarte, 2015. 
vo se constituye en una área de vacancia o a lo menos, muy poco explorado y trabajado por la investigación social en juventudes. Lo mismo ocurre en cuanto a investigación comparada con realidades juveniles de otros países.

Valparaíso, Chile. Agosto 2018.

\section{Referencias}

Abdala, E. (2002). Jóvenes, educación y empleo en América Latina. Papeles de Población, (33). México: uaemex.

Araujo, K. y Martuccelli, D. (2012). Desafíos comunes: retrato de la sociedad chilenay sus individuos. Santiago: Lom Ediciones.

Arriagada, I. (2004). Transformaciones sociales y demográficas de las familias latinoamericanas. Papeles de Población, (40). México: uaemex.

Baizán, P. (2006). El efecto del empleo, el paro y los contratos temporales en la baja fecundidad española de los años I99o. Revista Española de Investigaciones Sociológicas (REIS), (II5). Madrid: CIS.

Beck, U. (I998). La sociedad del riesgo. Hacia una nueva modernidad. Barcelona: Paidós.

Bendit, R. y Miranda, A. (20I7). La gramática de la juventud: un nuevo concepto en construcción. Última Década, (46). Santiago: Proyecto Juventudes.

Bourdieu, P. (ig88). La distinción. Criteriosy bases sociales del gusto. Madrid: Taurus.

Camarano, A. A. et al. (2004). Caminhos para a vida adulta: as múltiplas trajetórias dos jovens brasileiros. Texto para Discussão, (Iо38). Rio de Janeiro: IPEA.

Canales, M. y Ghiardo, F. (2012). Épocas, cohortes y generaciones de chilenos: exploración en torno a los grupos socioepocales. Última Década, (36). Valparaíso: Ediciones CIDPA.

Casal, J., García, M., Merino, R. y Quesada, M. (2006). Cambios en las modalidades de transición de los jóvenes los países del capitalismo informacional. Barcelona: Universidad Autónoma de Barcelona, Grup de Recerca Educació i Treball (GRET).

Corica, A. y Otero, A. (20I8).Transiciones juveniles: un análisis sobre el vínculo educación y trabajo de jóvenes egresados de la educación obligatoria argentina. Última Década, (48). Santiago: Proyecto Juventudes.

Cox, C. (2003). Las políticas educacionales de Chile en las últimas dos décadas del siglo XX. En: C. Cox (ed.), Políticas educacionales en el cambio de siglo. La reforma del sistema escolar en Chile. Santiago: Editorial Universitaria.

Dávila, O. y Ghiardo, F. (20I6). Procesos biográficos de la modernización en Chile. Polis, (44). Santiago: Universidad de Los Lagos. 
Dávila, O. y Ghiardo, F. (2012b). Transiciones a la vida adulta: generaciones y cambio social en Chile. Última Década, (37). Valparaíso: Ediciones cidPA.

Dávila, O. y Ghiardo, F. (2OIIc). Análisis comparativo a nivel nacional, descriptivo e interpretativo, de los resultados emanados de la Sexta Encuesta Nacional de fuventud, con respecto a las Encuestas Segunda, Tercera, Cuarta y Quinta de Fuventud. Santiago: Instituto Nacional de la Juventud (INJuv).

Dávila, O. y Ghiardo, F. (20IId). Trayectorias sociales juveniles: cursos y discursos sobre la integración la boral. Papers, g6 (4). Barcelona: Universidad Autónoma de Barcelona.

Dávila, O. y Ghiardo, F. (2008a). Trayectorias socialesjuveniles. Ambivalenciasy discursos sobre el trabajo. Valparaíso: Ediciones CIDPA e INJUv.

Dávila, O. y Ghiardo, F. y Medrano, C. (2005). Los desheredados. Trayectorias de viday nuevas condiciones juveniles. Valparaíso: Ediciones CIDPA.

De Oliveira, O. y García, B. (I990). Trabajo, fecundidad y condición femenina en México». Estudios Demográficosy Urbanos, I5 (5). México: El Colegio de México.

Duarte, C. (20I5). El adultocentrismo como paradigma y sistema de dominio. Análisis de la reproducción de imaginarios en la investigación social chilena sobre lo juvenil (tesis doctoral). Barcelona: Universidad Autónoma de Barcelona.

Elder, G. (I999). Children of the great depression: social change in life experience. Boulder, CO. Westview Press.

Featherman, D. y Sørensen, A. (1983). Societal Transformation in Norway and Change in the Life Course Transition into Adulthood. Acta Sociológica, (2).

Fuentes, A. et al. (2010). Postergación de la maternidad en Chile: una realidad oculta. Revista Médica de Chile, I38(ıо). Santiago: Sociedad Médica de Santiago.

Garretón, M. A. (1994). La faz sumergida del iceberg. Estudios sobre la transformación cultural. Santiago: CESOC y LOM Ediciones.

Ghiardo, F. y Canales, M. (20I8). Provincianos: modos de generación de la refundación agraria en Chile. En: A. Corica, A. Freytes y A. Miranda (comps.), Entrela educacióny el trabajo: la construcción cotidiana de las desigualdades juveniles en América Latina. Buenos Aires: CLACSO.

Giddens, A. (1998). La transformación de la intimidad. Sexualidad, amory erotismo en las sociedades modernas. Madrid: Editorial Cátedra.

Heinz, W. (2000). La transición de los jóvenes y el empleo en Alemania. Revista Internacional de la Ciencias Sociales, (I64). París: UnesCo.

Heinz, W. y Krüger, H. (200I). Life course: innovations and challenges for social research. Current Sociology, 49(2). Londres: Inglaterra.

Henríquez, H. y Uribe-Echeverría, V. (2003). Trayectorias laborales: la certeza de la incertidumbre. Cuadernos de Investigación, (I8). Santiago: Departamento de Estudios de la Dirección del Trabajo.

Henríquez, H., Rojas, M. L. y Díaz, C. (2004). Las mujeres en el empleo formal: diez años de investigación en Chile. Santiago: Universidad de Chile y Oxfam. 
Krüger, H. y Baldus, B. (I999). Work, Gender and the Life Course: Social Construction and Individual Experience. The Canadian Fournal of Sociology, $24(3)$.

Larrañaga, O., Cabezas, G. y Dussaillant, F. (20I3). Informe completo del estudio de la educación técnico profesional. Santiago: PNUD.

Lesthaeghe, R. y Moors, G. (200o). Recent Trends in Fertility and Household Formation in the Industrialized World. Review of Population and Social Policy, (9).

Longo, M. E. (2009). Género y trayectorias laborales. Un análisis del entramado permanente de exclusiones en el trabajo. Trayectorias, II(28). Nuevo León: Universidad Autónoma de Nuevo León.

Machado Pais, J. (2002). Laberintos de vida: paro juvenil y rutas de salida (jóvenes portugueses). Revista de Estudios de Fuventud, (56). Madrid: INJuvE.

Mannheim, K. (I982). O problema sociológico das gerações. En: M. Forachi, Mannheim. São Paulo: Ática.

Mannheim, K. (I944). Diagnóstico de nuestro tiempo. México: FCE.

Mayer, K. (2004). Life Courses and Life Chances in a Comparative Perspective. Yale University. Department of Sociology Center for Research on Inequalities and the Life Course And Max Planck Institute for Human Development, Berlin. Center of Sociology and the Study of the Life Course.

Mayer, K., Diewald, M. y Solga, H. (I993). Transitions to Post-Communism in East Germany: Worklife Mobility of Women and Men between I989 and I993. Acta Sociológica, 42(I).

Miranda, A. y Corica, A. (2018).Gramáticas de la juventud: reflexiones conceptuales a partir de estudios longitudinales en Argentina. En: A. Corica, A. Fuentes y A. Miranda (comps.), Entre la educacióny el trabajo: la construcción cotidiana de las desigualdades juveniles en América Latina. Buenos Aires: CLACSO.

Montiel, G. (2009). Transiciones virtuales en la juventud: una aproximación a la emancipación juvenil en la sociedad de la información. Recerca,(g).

Montilva, M. (2008). Postergación de la maternidad de mujeres profesionales jóvenes en dos metrópolis latinoamericanas. Utopíay Praxis Latinoamericana, (4I). Maracaibo: Universidad del Zulia.

Montilva, M. (2007). Individualización femenina y cohabitación sin papeles: el caso de las profesionales de Santiago de Chile. Kairos, (20). San Luis: Universidad Nacional de San Luis.

Mortimer, J. y Shanahan, M. (2003). Handbook of the Life Course. New York: Kluwer Acad.

Palma, I. (2012). Las nuevas generaciones de mujeres, el embarazo no previsto, las edades y la segmentación social en la sociedad chilena. En Revista Médica de Chile, I4o(3). Santiago: Sociedad Médica de Santiago.

Redondo, J. (2005). El experimento chileno en educación: ¿conduce a mayor equidad y calidad en la educación?. Última Década, (22). Valparaíso: 
Ediciones CIDPA.

Rodríguez, J. (2005). Reproducción en la adolescencia: el caso de Chile y sus implicaciones de política. Revista de la CEPAL No86. Santiago: CEPAL.

Schkolnik, S. y Chakiel, J. (2004). Los sectores rezagados en la transición de la fecundidad en América Latina. Revista de la CEPAL, (83). Santiago: CEPAL.

Sepúlveda, L. (20ı0). Las trayectorias de vida y el análisis de curso de vida como fuentes de conocimiento y orientación de políticas sociales. Revista Perspectivas de Trabajo Social, (2I). Santiago: Departamento de Trabajo Social, Universidad Católica Silva Henríquez.

Torche, F. y Wormald, G. (2004). Estratificación y movilidad social en Chile: entre la adscripción y el logro. Serie Políticas Sociales, (98). Santiago: CEPAL.

Vaan de Kaa, D. (ig87). Europe's Second Demographic Transition. Population Bulletin, (42).

Van de Velde, C. (2005). La entrada en la vida adulta. Una comparación europea. Revista de Estudios de Fuventud, (7I). Madrid: InJuve.

Villalón, G. y Vera, S. (20I2). Panorama demográfico en Chile contemporáneo: desafíos para la sociedad del siglo xxi. Revista Anales de la Universidad de Chile, Séptima Serie, (3). Santiago: Universidad de Chile.

Weller, J. (2006) (ed.). Los jóvenes y el empleo en América Latina desafíos y perspectivas ante el nuevo escenario laboral. Santiago: CEPAL.

Zilvetti, M. (2009). Dimensiones socioculturales implicadas en la no-inserción laboral juvenil. Observatorio de fuventud, (21). Santiago: Instituto Nacional de la Juventud. 